\title{
Regulation of Reversible Dissociation of LHCII from PSII by Phosphorylation in Plants
}

\author{
Zhenhai Cui $^{1,2}$, Yanpeng Wang ${ }^{1}$, Ao Zhang ${ }^{1}$, Lijun Zhang ${ }^{1,2 *}$ \\ ${ }^{1}$ Liaoning Province Research Center of Plant Genetic Engineering Technology, Shenyang, China; ${ }^{2}$ Biological Science and Technol- \\ ogy College, Shenyang Agricultural University, Shenyang, China. \\ Email: "lijunzhang8@aliyun.com
}

Received November $26^{\text {th }}, 2013$; revised December $27^{\text {th }}, 2013$; accepted January $16^{\text {th }}, 2014$

Copyright (c) 2014 Zhenhai Cui et al. This is an open access article distributed under the Creative Commons Attribution License, which permits unrestricted use, distribution, and reproduction in any medium, provided the original work is properly cited. In accordance of the Creative Commons Attribution License all Copyrights @ 2014 are reserved for SCIRP and the owner of the intellectual property Zhenhai Cui et al. All Copyright (C) 2014 are guarded by low and by SCIRP as a guardian.

\section{ABSTRACT}

LHCII is a crucial light-harvesting pigment/protein complex in photosystem II (PSII) supercomplex. It also participates in the light energy redistribution between photosystems and in the photoprotection via its reversible dissociation with PSII and PSI (photosystem I). This reversible detachment of LHCII is regulated by phosphorylation of its own and PSII core protein. Under low light conditions, LHCII is phosphorylated and dissociated with PSII core protein complex and combined with PSI, which balances the excitation energy between PSII and PSI; Under high light environment, the phosphorylation of PSII core proteins makes LHCII detach from PSII. The dissociated LHCII presents in a free state, which involves in the thermal dissipation of excess excitation energy. During photodamage, dual phosphorylations of both PSII core proteins and LHCII complexes occur. The phosphorylation of D1 is conductive to the disintegration of photodamaged PSII and the cycle of repair. In this circumstance, the phosphorylation of LHCII is induced by reactive oxygen species (ROS) and then the phosphorylated LHCII migrates to PSI, into the repair cycle of damaged PSII. The ferredoxin (Fdr) and thioredoxin (Tdr) system may play a possible central role in the phosphorylation regulation on LHCII dissociation.

\section{KEYWORDS}

Light-Harvesting Complex II (LHCII); Phosphorylation; Photosystems; Photoinhibition; Ferredoxin and Thioredoxin System

\section{Introduction}

Photosynthetic organisms utilize sunlight energy, $\mathrm{CO}_{2}$ and $\mathrm{H}_{2} \mathrm{O}$ to synthesize carbohydrates. The photosynthetic process consists of light reaction and dark reaction. In plants, light reaction occurs inside chloroplasts, to generate NADPH and ATP by photosynthetic electron transfer chain and photophosphorylation. The resulting NADPH and ATP are used to converse carbon dioxide into carbohydrates in dark reaction. The electron transfer chain locates in the membrane of thylakoid, containing the two types of photosystems (PSII, PSI) and cytochrome b6-f (Cytb6-f) complexes. NADP production depends on the linear electron transfer involving PSII, $\mathrm{Cytb}_{6} \mathrm{f}$ and PSI. Extra ATP is synthesized by the cyclic electron transfer

\footnotetext{
*Corresponding author.
}

only involving PSI and $\mathrm{Cytb}_{6}-\mathrm{f}$. There is a difference in light absorption wavelength between PSII and PSI. With these characteristics of photosynthesis, to acquire the high efficiency of light utilization, plants not only need to coordinate the action between light reaction and dark reaction, but also between PSII and PSI.

Light is the sole energy for plant photosynthesis, but it also damages the photosynthetic apparatus [1-4]. Under fluctuating light or strong light conditions, the imbalance allocation of excitation energy occurs between PSII and PSI, or the captured energy in light reaction beyond the need of dark reaction. The resulting excess energy in photosynthetic apparatus damages PSII complexes and reduces the photosynthetic efficiency. This phenomenon is so-called photoinhibition or photodamage. In order to avoid this injury, plants have developed a series of specific 
protection mechanism in chloroplasts during the longterm evolution [5-7]. This mechanism involves the architectural shifts in PSII complexes and thylakoid membrane [8]. One of them is the reversible detachment of light-harvesting pigment complex with PSII and PSI core complex [9-11]. Under the light condition with changing wavelength, the reversible detachment of LHCII complex makes the excitation energy redistributed between the two photosystems $[10,12]$. In continuous intensive light condition, a number of LHCIIs dissociate from PSII and present in a free state inside chloroplast stroma, preventing more excitation energy from destroying the reaction centers $[13,14]$. Several reports show that the detachment of LHCII also participates in PSIIrepair mechanism during photodamage $[15,16]$. In higher plants, the reversible dissociation of LHCII with the photosystems is regulated by phosphorylation $[6,17]$, and the mechanism of phosphorylation according to the light intensity.

\section{LHCII Phosphorylation in State Transition}

The reaction centers of PSII and PSI have different light absorption characteristics, their absorption peak, respectively, at $680 \mathrm{~nm}$ and $700 \mathrm{~nm}$. As green plants are subjected to light environment with fluctuating intensity, PSII and PSI are excited in out-balance and LHCIIs displaced from the over-excitation photosystems to the lessexcitation photosystem, which is so-called state transition $[12,18]$. Under photoinhibition conditions, state transition is also considered as a very important photoprotection mechanism of PSII [19].

The reversible phosphorylation of LHCII complex regulates the reversible displacement of LHCII between two photosystems is regulated by the phosphorylation/ dephosphorylation of LHCII complex [17], because the phosphorylation state of LHCIIchanges LHC II affinity with the two different photosystems [6]. As PSII complex is overly excited, LHCII is phosphorylated. And then the phosphorylated LHCII dissocates from PSII to move towards PSI, which is called state 2. When PSI is excessively excited, the phosphorylated LHCII is dephosphorylated and detached from PSI and then returns to PSII, which is called state $1[11,20]$. In unicellular organisms, the mobile fraction of LHCII during state transitions is approximately $80 \%$, whereas in land plants the percent is only $15 \%-20 \%$ [11].

The major function of photosynthetic electron transport chain is to synthesize NADPH and ATP. In some circumstances, plants need to strengthen cyclic photosynthetic electron transfer to generate more ATP. State transitions are found to participate in the regulation of ATP synthesis. Owens and Ohad (1982) [21] restricted ATP synthesis in Chlamydomonas reinhardtii by inhi- biting chloroplast respiration, the phosphorylation level of LHCII significantly increased and accompanied by $\mathrm{cytb}_{6}-\mathrm{f}$ complex movement to PSI, which promoted the cyclic electron transfer and ATP generation. The similar phenomenon was observed in higher plants. The inhibition of ATP synthesis in tobacco plants induced the state transition and also accelerated the cyclic electron flow [22].

PSII is a dimeric supercomplex containing changeable number of outer LHCII complexes [23]. The LHC II consists of trimeric LHCII complexes, from 2 to 4 copies [24]. The polypeptides are encoded by the genes of lhcb1, lhcb2 and lhcb3 [25]. Lhcb1 and Lhcb2 have been well documented to reversibly phosphorylated/dephosphorylated, and Lhcb3 has not been found to have any phosphorylation site. After phosphorylated and dissociated from PSII, LHCII trimer is depolymerized and releases phosphorylated monomers that binds to PSI [10]. The connection between LHCII antenna and PSII core complex is through antenna proteins of CP29, CP26 and CP24, which are the products of genes of lhcb4, lhcb5 and lhcb6 [24,26,27]. In Arabidopsis, three isoforms of CP29 are found, named as Lhcb4.1, Lhcb4.2 and Lhcb4.3 [28]. Nevertheless, the difference in function of these protein isoforms has remained elusive.

The recent studies have revealed the protein kinases involved in PSII phosphorylation events [29]. In Arabidopsis, two orthologue thylakoid-associated serine/ threonine kinases, called STN7 and STT7, were identified by mutant analysis approach and found to be the LHCII phosphokinase [6,9]. They are single-spanning membrane domain proteins. Their $\mathrm{N}$-terminal regions locate in luminal side and C-terminal kinase domains in stromal side in cloroplasts. STN7 is essential for reversible LHCII polypeptide phosphorylation. This phosphorylation is required for state transitions in Arabidopsis [30-33]. The loss of STN7 in plants blocks the movement of LHCII complex from PSII core complex to PSI core complex $[30,34]$. Low light intensity activates STN7, but high light intensity deactivates it $[31,35]$. The redox state of mobile electron carriers between photosystems such as plastoquinone (PQH2 and $\mathrm{PQ}$ ) [35-39] and ferredoxin/ thioredoxin system [12] regulate the activity of STN7. The lack of PQH2 at the Qo site inside Cytb6-f complex results in STN7 deactivation in dark. Under short-wavelength light, $\mathrm{PSII}$ is over-excited, $\mathrm{PQH}_{2}$ binding to the Qo site and causing a conformational change in $\mathrm{Cytb}_{6}-\mathrm{f}$, which transfers the reduction signal from thylakoid lumen to the kinase domain in stromal side and activates LHCII kinase, resulting in LHCII phosphorylation [15, 40]. A new model is proposed by Puthiyaveetil (2011) [12]. In his model, when PSII is overly excited, PQH2 released by Qo site reduces a disulfide bond between conserved luminal cysteine residues in STN7 and the 
kinase is activated. Under strong light, STN7 is inactivated via the ferredoxin/thioredoxin system by the reduction of stromal disulfide bond. This model better explained the inactivation of STN7. Moreover, we consider that the ferredoxin/thioredoxin system regulates both STN7 and phosphatase PPH1/TAP38 activity. STN7 can catalyze the phosphorylation of D1, D2 and CP43 of PSII core complex, but in a very low level.

CP29 and TSP9, the linker proteins [41,42] regulate state transitions by their differential phosphorylation [6, $43,44]$. Under strong light condition, stn8 and wild type Arabidopsis thaliana selectively increased the phosphorylation of CP29 isoforms Lhcb4.1 and Lhcb4.2 at threonine residues in five different positions, whereas in $\operatorname{stn} 7$ and $\operatorname{stn} 7 / \operatorname{stn} 8$ palnt, the four phosphorylation positions were not found [45].

The dephosphorylations of Lhcb1 and Lhcb2 are majorly catalyzed by PPH1/TAP38 protein phosphatase. In mutants with loss of the activity of PPH1/TAP38, the size of PSI light harvesting complex grew and state transition1 is inhibited [46,47]. It is suggested that when PSI is over excited under long-wavelength light, $\mathrm{PQH} 2$ is oxidized and released from the Qo site, and the phosphatases in stromal side become active and catalyze the dephosphorylation of phosphorylated LHCIIproteins [48, 49], LHCII dissociated from PSII.

When state 1 transition occurs, the phosphorylated LHCII is dephosphorylated and detached from PSI and then returns to PSII. For maintaining this process, it is essential for photosynthetic systems to enhance dephosphorylation reaction and inhibit phosphorylation reaction of LHCII. We propose that the ferredoxin/thioredoxin system plays a central role in this process (as shown in Figure 1). While overly excited, PSI generates more reduced ferredoxin (Fdr) and thioredoxin (Tdr), which activates the phosphatase PPH1/TAP38 and inactivates

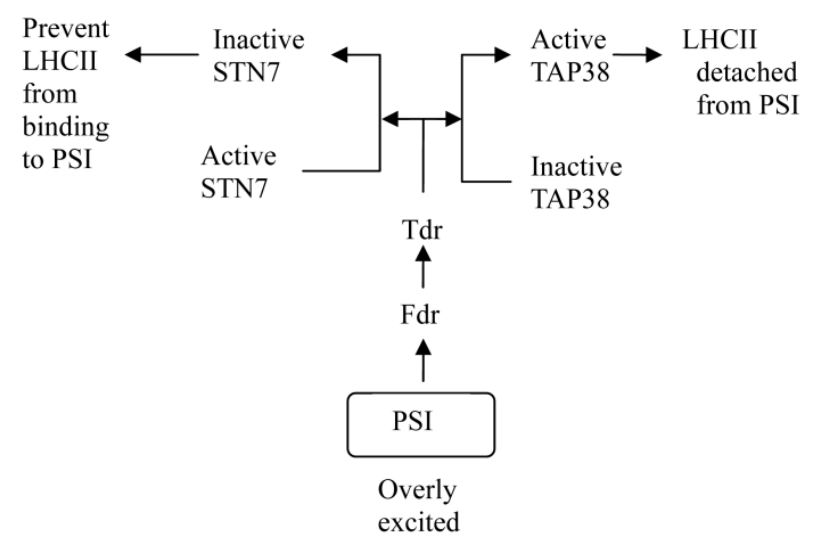

Figure 1. A diagram of the ferredoxin and thioredoxin regulation on STN7 and TAP38 during state 1 transition. When PSI is overly excited and generates more reduced ferredoxin (Fdr) and thioredoxin (Tdr), which activate the phosphatase PPH1/TAP38 and inactivate STN7.
STN7 via the reduction of stromal disulfide linkage [12].

\section{Phosphorylation of PSII Core Proteins in Photoprotection}

The reversible dissociation of LHCII from PSII also occurs under high light intensity, but the mechanism differs from that in low light condition. The phosphorylation of core proteins in PSII complex occurs during photoprotection. The original and primary site of photodamage is locked in PSII [1]. In order to avoid this injury, plants need decreasing the excitation energy transferred to PSII reaction centers in high light condition. Therefore, LHCII detachment from PSII and PSI is conductive to this energy-decreasing process. But, under such high light condition, the phosphorylation of LHCII is restricted [20]. Several studies have shown that the ferredoxin and thioredoxin system at the side of PSI the down regulated the LHCII phosphorylation. The protein kinase STN7, which catalyzes LHCII phosphorylation, has disulfide bonds on the luminal side [17]. The disulfide bonds may be reduced to sulfhydryl group by ferredoxin-thioredoxin system, resulting in STN7 inactivation [12]. However, a fraction of LHCII are still detached from PSII under strong light condition, and the detached LHCIIs present in a free state, not binding to PSI [14]. The free LHCIIs dissipate the excitation energy via fluorescence release and thermal radiation. This process prevents the excitation energy which is not needed by reaction centers, from entering the reaction centers [14,50,51].

The protein phosphorylation of PSII core complex regulates the detachment of LHCII from PSII under strong light, when the phosphorylation of LHCII proteins is restricted. D1, D2, CP43 and PsbH of PSII core proteins were phosphorylated under high light intensity [14, 16,17]. PSII was overexcited in the mutants with loss of STN7 kinase under low intensity of light, but the overexcitation was not found under strong light. The mutant analyses on Arabidophsis indicate that the core proteins of PSII is phosphorylated by the protein kinase STN8, rather specific for these proteins [52,53]. This kinase is different from STN7 in structure. It has no disulfide bond in the luminal side so that the activity is not inhibited by ferredoxin-thioredoxin system. Consequently, core proteins of PSII complex are still phosphorylated by high irradiance condition. Zhang and Xu (2003) [14] found the core protein phosphorylation of PSII can induce the changes in the structure of PSII reaction center. These changes may loose the bounding of LHCII with PSII reaction center complex, in favor of LHCII dissociation. When green plants are moved to low intensity of light or dark condition, D1 protein is dephosphorylated by phosphatase [54], LHCII returning to PSII again. This phosphatase localizes in stacked thylakoid membrane and catalyzes the dephosphorylation of D1 in functional PSII 
centers, independent of light, which is different from the phosphatase functioning in PSII repair [55]. In a reverse genetic screen, a PSII core protein phosphatase (PBPC) was identified, which counteracts the activity of STN8 kinases and is required for efficient dephosphorylation of PSII core proteins [56,57].

During high light, PSII and PSI need to prevent the accumulation of excess light energy. The detached LHCII must be prevented from phosphorylation again and blocked to bind PSI. The phosphorylated D1 proteins need to remain in phosphorylation state during high light. In this process, the ferredoxin/thioredoxin system may be a central coordinator (as shown in Figure 2). Under high light, PSII through PSI generates more reduced ferredoxin (Fdr) and thioredoxin (Tdr), which activate STN8 and inactivate PSII core protein phosphatase (PBPC) possibly by kinases. STN7 is also inhibited and prevented to phosphorylate LHCII, not associating with PSI. In spite of lacking of disulfide bond, STN8 activity is not inhibited by ferredoxin-thioredoxin system [52,53], but it may be regulated by phosphorylation [58].

\section{LHCII Phosphorylation in Photodamage}

In high light intensity, LHCII separates from PSII via the core protein phosphorylation of PSII in plants, and is avoided combining with PSI to prevent photoinhibition, even photodamage [3]. However, a number of studies have shown that, when plants are subjected to strong light condition, the phosphorylation of a fraction of LHCII complex remains to occur $[16,39]$. This phosphorylation may be due to the production of reactive oxygen species (ROS) in PSII reaction center. ROS serves as an oxidant that damages the PSII reaction centers and oxi-

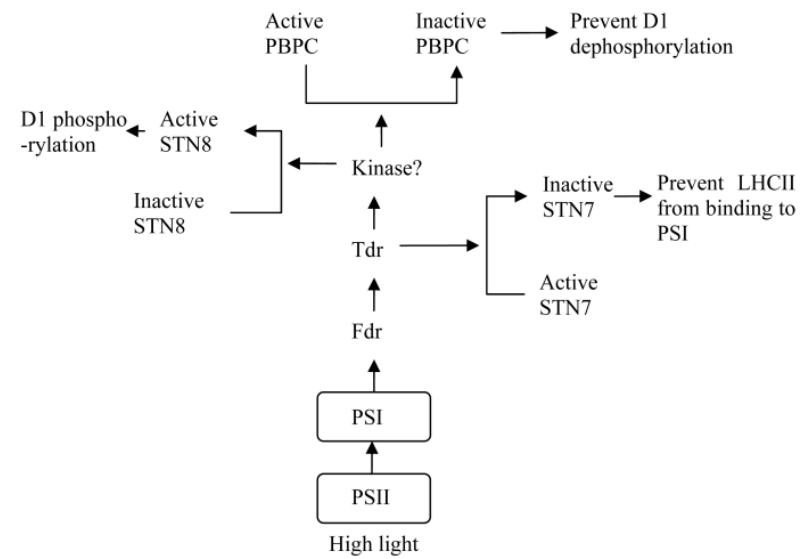

Figure 2. A diagram of the ferredoxin and thioredoxin regulation on STN8, PBPC and STN7 during high light. Under high light, PSII through PSI generates more reduced ferredoxin (Fdr) and thioredoxin (Tdr), which activate STN8 and inactivate PSII core protein phosphatase (PBPC) possibly by kinases. STN7 is also inhibited and prevented to phosphorylate LHCII. dizes the sulfhydryl group to the disulfide bond of the inactivated LHCII kinase STN7 in thylakoids membrane, restoring the catalytic activity of the enzyme. Breitholtz et al. (2005) [39] found that, under high light intensity, in both Arabidopsis plants with higher excitation capacity of PSII and with defectiveness in non-photochemical reaction activity, the phosphorylation in LHCII occurred. Because the lower ability of non-photochemical quenching and higher excitation capacity of PSII result in more excess energy accumulation in PSII reaction centers, and the generating hydrogen peroxide and singlet oxygen restored the catalytic activity of the LHCII kinase. As a result, the phosphorylated LHCII migrates to the PSI enriched stromal region from the PSII enriched granal region of the thylakoid membrane and transfers the excitation energy to PSI [16]. Then, what function is the binding of LHCII to PSI and whether the binding leads to the damage to PSI?

The phosphorylation of LHCII induced by the high light intensity may involve in repair mechanism of PSII [15]. Of the core proteins of PSII complex, D1 is susceptible to be injured and the turnover rate is fast. Under prolonged high irradiance, ROS generated by PSII reaction center, can inevitably damage the D1 protein [59]. The rate of photodamage increases as irradiance intensity rises. On the other hand, the newly synthesized functional D1 displaced the injured one in PSII core complex and the repair kept pace with the damage. Otherwise, the structure and function of photosynthetic apparatus were severely disrupted. The repair process proposed includes: 1) disassembly of injured PSII-LHCII supercomplex into PSII and LHCII, and PSII core dimer into monomer; 2) movement of the PSII monomer from the stacked membrane region of thylokoid to the stroma region of nonstacked membrane; 3) D1, D2 and CP43 proteins were dephosphorylated; 4) degradation of injured D1 protein; 5) the newly biosynthesized D1 protein cotranslationally inserted PSII complex [60]. The repair cycle of inactivated PSII is associated with dephosphorylation of phosphorylated and photodamaged D1 and is a light-dependent reaction in vivo [55].

The PSII repair rate was decreased while the generation of ATP was blocked in Spirodela oligorrhiza [61,62] because the synthesis of ATP is indispensable for the D1 protein synthesis. The binding of phosphorylated LHCII to PSI accelerates the cyclic electron transfer and enhances the synthesis of ATP [21]. Consequently, the phosphorylation of LHCII is related to the transient repair of damaged PSII complex in photoinhibition. Murata et al. (2007) [62] proposed in his review that unfavorable environmental factors other than high light majorly inhibited the PSI repair cycle. For instance, high salt, low $\mathrm{CO}_{2}$, low temperature and mild heat stress all blocked de novo synthesis of proteins. 
During the photodamage, PSII core proteins are also phosphory lated like LHCII complex. These dual phosphorylations may more conductive to the repair of the injured PSII. Within chloroplasts, strong light causes the excitation imbalance between PSII and PSI leading to ROS generation and D1 photodamage. Plants prevent and recover from this damage by rearranging the structure of photosynthetic membrane [63]. The phosphorylation of PSII core polypeptides is of importance when the PSII core complex is rapidly photodamaged during high light stress [64]. The movement of impaired PSII complex from PSII-enriched regions to PSI-enriched regions for restore is facilitated by the core protein phosphorylation. A hypothesis supposed that the phosphorylation of injured D1 protein was a signal for the PSII complex movement to PSI-enriched regions from PSII-enriched regions $[65,66]$. In the PSI-enriched regions, proteases DegP and FtsH decomposed the damaged D1 protein $[65,66]$. In light condition, the decomposition of damaged D1 protein is indeed delayed in the mutants of stn 8 and $\operatorname{stn} 7 / \operatorname{stn} 8$, other than in the plants of wild type and stn7 [30]. The phosphorylation of PSII core protein participates in the change of thylakoid membrane contexture, which facilitated the transport of damaged PSII complex to the PSI-enriched regions for repair. Lack of STN8 impeded the shift of the stacked thylakoid membrane to non-stacked membrane [67]. The stack of photosynthetic membrane blocks the lateral displacement of thylakoid proteins and restrains the conversion of D1 proteins damaged by strong irradiance [67-69]. The stack of thylakoid membrane was not influenced by the lack of STN7 activity [30]. On the contrary, the translocation of movable LHCII fraction from PSII to PSI and state transition1 were impeded $[42,34]$. The release of PSII subunits was partially regulated by phosphorylation in PSII membranes [70,71]. Upon the phosphorylation of the PSII core proteins, the thylakoid membrane architecture was switched to a state with more fluid, as a result, promoting the cycle of PSII complex repair [72]. In summary, the dual phosphorylations of PSII core proteins and LHCII complexes enhance the dissociation of damaged PSII core protein with LHCII complexes and the migration of the PSII to stromal regions, via the conformational change of thylakoid membrane.

During photodamage occurs, chloroplasts not only need to prevent the accumulation of excess excitation energy in PSII, but also to repair the damaged PSII (as shown in Figure 3). In photodamage, PSII through PSI generates more reduced ferredoxin (Fdr) and thioredoxin (Tdr), which, possibly via kinase, activate STN8 and inactivate PSII core protein phosphatase (PBPC) in PSII membrane region and activates the inactive phosphatase in PSI membrane for D1 dephosphorylation and repair. STN8 catalyseds D1 protein phosphorylation is neces-

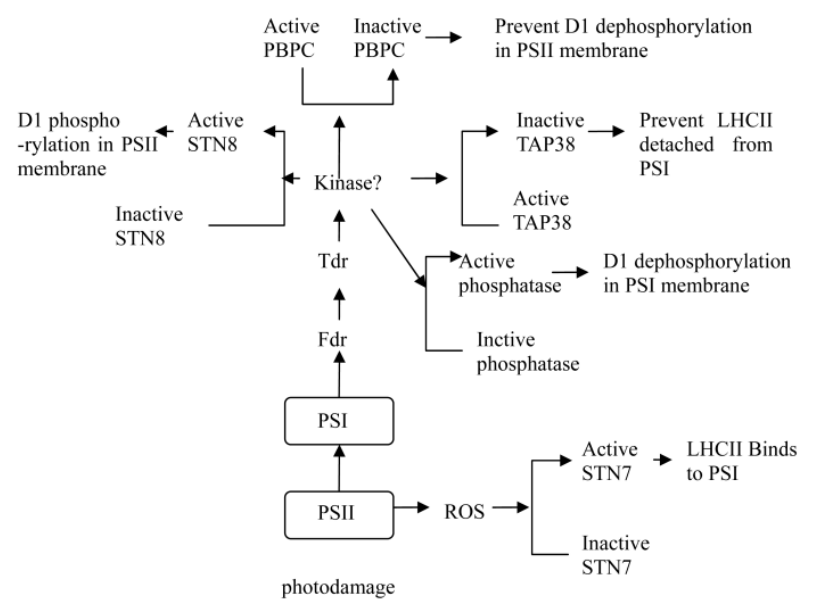

Figure 3. A diagram of the ferredoxin and thioredoxin regulation on STN8, PBPC and STN7 under photodamage. When photodamage occurs, PSII produces reactive oxygen species (ROS) and through PSI generates more reduced ferredoxin (Fdr) and thioredoxin (Tdr). Fdr/Tdr activates STN8 and inactivates PSII core protein phosphatase (PBPC) in PSII membrane region possibly by kinases. The sulfhydryl group to the disulfide bond oxidized by ROS, the inactivated LHCII kinase STN7 become active. Active TAP38 may be inactivated via kinase (different from its activation). In addition, the inactive phospatase in PSI membrane may be activated by Fdr/Tdr system via kinase for D1 dephosphorylation and repair.

sary for PSII repair, because damaged PSII repair depends on the dissociation of complexes. D1 phosphorylation is conductive to prevent excitation energy accumulation in PSII. In addition, under photodamage, PSII produces reactive oxygen species (ROS), oxidizing the sulfhydryl group to the disulfide bond and activates the inactivated LHCII kinase STN7. LHCII phosphorylation is not only beneficial to the disassembly of PSII complexes, but also enhances cyclic electron transport to synthesize ATP needed to repair damaged PSII complex. Active TAP38 may be inactivated via kinase, which is different from its activation.

\section{Perspectives}

Recent studies have shown that the regulation of reversible dissociation of LHCII is closely related to the site and amount of phosphorylation, but less is known about how plants differentially recognize the sites and control the amount. The regulation of light intensity to this process may be in a more complicated mechanism. In addition, during photodamage, the phosphorylated LHCII binds to PSI reaction center and transfers extra excitation energy to it, a potential damage. Therefore, we need more information about the mechanism of photoprotection of PSI reaction centers in such circumstance. And we know that the low light intensity condition is in favor of the repair of photodamaged PSII. This repair process, in- 
volving the synthesis of $\mathrm{D} 1$ proteins de novo, needs extra ATP. But the low light intensity also activates phosphatases, resulting in the dephosphorylation of LHCII and the dissociation from PSII. As a result, the cyclic electron transfer and the synthesis of ATP will be decreased. Therefore, plants need a complicated system to ensure the process of D1 repair. Evidence shows that the phosphoryltion regulation of reversible dissociation of LHCII with photosystems at least consists of ferredoxin/thioredoxin system and kinase system. Up to date, we have known little about kinases in this regulation network.

\section{Acknowledgements}

The study is supported by the National Natural Science Foundation of China (No. 31000673, 31370283, 31070225, 31201141 and 31070157), Ministry of National Education Doctoral Fund of China (20102103110001).

\section{REFERENCES}

[1] E. Hideg and N. Murata, "The Irreversible Photoinhibition of the Photosystem II Complex in Leaves of Vicia faba under Strong Light,” Plant Science, Vol. 130, No. 2, 1997, pp. 151-158. http://dx.doi.org/10.1016/S0168-9452(97)00227-6

[2] Z. L. Hu, Z. H. Cui, P. Qin, S. Y. Cao, L. J. Zhang and G. Z. Zhou, "Excess Light Energy Dissipation and Photooxidation Defence of Chinese Pine Needles During the Late Winter," Journal of Shenyang Agricultural University (China), Vol. 43, No. 5, 2012, pp. 555-559.

[3] S. Takahashi and N. Murata, "How Do Environmental Stresses Accelerate Photoinhibition?" Trends in Plant Science, Vol. 13, No. 4, 2008, pp. 178-182. http://dx.doi.org/10.1016/j.tplants.2008.01.005

[4] Y. P. Wang, Z. H. Cui, Y. S. Zhu, J. J. Fan and L. J. Zhang, "The Comparison of Anatomic Structure and Photoinhibition Characteristics between Different Regions of the C4 Photosynthetic Leaf in Maize (Zea mays L.),” Plant Physiology Journal (China), Vol. 48, No. 6, 2012, pp. 571-576.

[5] C. Critchley and A. W. Russell, "Photoinhibition of Photosynthesis in Vivo: The Role of Protein Turnover in Photosystem II,” Physiologia Plantarum, Vol. 92, No. 1, 1994, pp. 188-196. http://dx.doi.org/10.1111/j.1399-3054.1994.tb06670.x

[6] J. Kargul and J. Barber, "Photosynthetic Acclimation: Structural Reorganisation of Light Harvesting AntennaRole of Redox-Dependent Phosphorylation of Major and Minor Chlorophyll a/b Binding Proteins,” FEBS Journal, Vol. 275, No. 6, 2008, pp. 1056-1068.

http://dx.doi.org/10.1111/j.1742-4658.2008.06262.x

[7] G. R. Cui , L. J. Zhang, Y. S. Zhu, Z. H. Cui and Z. H. Li, "Formation of Plant C4 Photosynthetic Pathway and Its Influencing Factors," Plant Physiology Communications (China), Vol. 7, 2009, pp. 711-720.

[8] H. Kirchhoff, "Architectural Switches in Plant Thylakoid Membranes,” Photosynthesis Research, Vol. 116, No. 2-3,
2013, pp. 481-487.

http://dx.doi.org/10.1007/s11120-013-9843-0

[9] A. Haldrup, P. E. Jensen, C. Lunde and H. V. Scheller, "Balance of Power: A View of the Mechanism of Photosynthetic State Transitions," Trends in Plant Science, Vol. 6, No. 7, 2001, pp. 301-305.

http://dx.doi.org/10.1016/S1360-1385(01)01953-7

[10] J. F. Allen, "State Transitions-A Question of Balance," Science, Vol. 299, No. 5612, 2003, pp. 1530-1532. http://dx.doi.org/10.1126/science.1082833

[11] L. Dietzel, K. Bräutigam and T. Pfannschmidt, "Photosynthetic Acclimation: State Transitions and Adjustment of Photosystem Stoichiometry-Functional Relationships between Short-Term and Long-Term Light Quality Acclimation in Plants,” FEBS Journal, Vol. 275, No. 6, 2008, pp. 1080-1088. http://dx.doi.org/10.1111/j.1742-4658.2008.06264.x

[12] S. Puthiyaveetil, "A Mechanism for Regulation of Chloroplast LHC II Kinase by Plastoquinol and Thioredoxin,” FEBS Letters, Vol. 585, No. 12, 2011, pp. 1717-1721. http://dx.doi.org/10.1016/j.febslet.2011.04.076

[13] R. G. Walters and P. Horton, "Resolution of Components of Non-Photochemical Chlorophyll Fluorescence Quenching in Barley Leaves,” Photosynthesis Research, Vol. 27, No. 2, 1991, pp. 121-133. http://dx.doi.org/10.1007/BF00033251

[14] H. B. Zhang and D. Q. Xu, "Role of Light-Harvesting Complex 2 Dissociation in Protecting the Photosystem 2 Reaction Centres against Photodamage in Soybean Leaves and Thylakoids,” Photosynthetica, Vol. 41, No. 3, 2003, pp. 383-391.

http://dx.doi.org/10.1023/B:PHOT.0000015462.71601.d7

[15] O. Kruse, "Light-Induced Short-Term Adaptation Mechanisms under Redox Control in the PSII-LHCII Supercomplex: LHC II State Transitions and PS II Repair Cycle,” Naturwissenschaften, Vol. 88, No. 7, 2001, pp. 284-292. http://dx.doi.org/10.1007/s001140100232

[16] P. Martinsuo, S. Pursiheimo, E. M. Aro and E. Rintamäki, "Dithiol Oxidant and Disulfide Reductant Dynamically Regulate the Phosphorylation of Light-Harvesting Complex II Proteins in Thylakoid Membranes," Plant Physiology, Vol. 133, No. 1, 2003, pp. 37-46. http://dx.doi.org/10.1104/pp.103.027268

[17] V. Bonardi, P. Pesaresi, T. Becker, E. Schleiff, R. Wagner, T. Pfannschmidt, P. Jahns and D. Leister, "Photosystem II Core Phosphorylation and Photosynthetic Acclimation Require Two Different Protein Kinases,” Nature, Vol. 437, No. 7062, 2005, pp. 1179-1182. http://dx.doi.org/10.1038/nature04016

[18] T. Mikko, M. Piippo, M. Suorsa, S. Sirpiö, P. Mulo, J. Vainonen, A. V. Vener, Y. Allahverdiyeva and E. M. Aro, "State Transitions Revisited-A Buffering System for Dynamic Low Light Acclimation of Arabidopsis," Plant Molecular Biology, Vol. 62, No. 4-5, 2006, pp. 779-793. http://dx.doi.org/10.1007/s11103-006-9044-8

[19] C. W. Mullineaux, E. Bittersmann, J. F. Allen and A. R. Holzwarth, "Picosecond Time-Resolved Fluorescence Emission Spectra Indicate Decreased Energy Transfer from the Phycobilisome to Photosystem II in Light-State 
2 in the Cyanobacterium Synechococcus 6301," Biochimica et Biophysica Acta (BBA)—Bioenergetics, Vol. 1015, No. 2, 1990, pp. 231-242. http://dx.doi.org/10.1016/0005-2728(90)90025-Y

[20] F. A. Wollman, "State Transitions Reveal the Dynamics and Flexibility of the Photosynthetic Apparatus," Embo Journal, Vol. 20, No. 14, 2001, pp. 3623-3630. http://dx.doi.org/10.1093/emboj/20.14.3623

[21] G. C. Owens and I. Ohad, "Phosphorylation of Chlamydomonas reinhardi Chloroplast Membrane Proteins in Vivo and in Vitro," The Journal of Cell Biology, Vol. 93, No. 3, 1982, pp. 712-718. http://dx.doi.org/10.1083/jcb.93.3.712

[22] M. Rott, N. F. Martins, W. Thiele, W. Lein, R. Bock, D. M. Kramer and M. A. Schöttler, "ATP Synthase Repression in Tobacco Restricts Photosynthetic Electron Transport, $\mathrm{CO}_{2}$ Assimilation, and Plant Growth by Overacidification of the Thylakoid Lumen,” Plant Cell, Vol. 23, No. 1, 2011, pp. 304-321. http://dx.doi.org/10.1105/tpc.110.079111

[23] Z. Liu, H. Yan, K. Wang, T. Kuang, J. Zhang, L. Gui, X. An and W. Chang, "Crystal Structure of Spinach Major Light-Harvesting Complex at 2.72 Å Resolution,” Nature, Vol. 428, No. 6980, 2004, pp. 287-292. http://dx.doi.org/10.1038/nature02373

[24] J. P. Dekker and E. J. Boekema, "Supramolecular Organization of Thylakoid Membrane Proteins in Green Plants," Biochimica et Biophysica Acta (BBA)—Bioenergetics, Vol. 1706, No. 1-2, 2005, pp. 12-39.

http://dx.doi.org/10.1016/j.bbabio.2004.09.009

[25] S. Caffarri, R. Croce, L. Cattivelli and R. Bassi, “A Look within LHCII: Differential Analysis of the Lhcb1-3 Complexes Building the Major Trimeric Antenna Complex of Higher-Plant Photosynthesis,” Biochemistry, Vol. 43, No. 29, 2004, pp. 9467-9476.

[26] A. E. Yakushevska, W. Keegstra, E. J. Boekema, J. P. Dekker, J. Andersson, S. Jansson, A. V. Ruban and P. Horton, "The Structure of Photosystem II in Arabidopsis: Localization of the CP26 and CP29 Antenna Complexes," Biochemistry, Vol. 42, No. 3, 2003, pp. 608-613. http://dx.doi.org/10.1021/bi027109z

[27] B. van Oort, M. Alberts, S. de Bianchi, L. Dall'Osto, R. Bassi, G. Trinkunas, R. Croce and H. van Amerongen, "Effect of Antenna-Depletion in Photosystem II on Excitation Energy Transfer in Arabidopsis thaliana," Biophysical Journal, Vol. 98, No. 5, 2010, pp. 922-931. http://dx.doi.org/10.1016/j.bpj.2009.11.012

[28] B. Zybailov, H. Rutschow, G. Friso, A. Rudella, O. Emanuelsson, Q. Sun and K. J. van Wijk, "Sorting Signals, N-Terminal Modifications and Abundance of the Chloroplast Proteome,” PLoS One, Vol. 3, No. 4, 2008, p. e1994.

[29] S. Reiland, G. Messerli, K. Baerenfaller, B. Gerrits, A. Endler, J. Grossmann, W. Gruissem and S. Baginsky, "Large-Scale Arabidopsis Phosphoproteome Profiling Reveals Novel Chloroplast Kinase Substrates and Phosphorylation Networks," Plant Physiology, Vol. 150, No. 2, 2009, pp. 889-903.

http://dx.doi.org/10.1104/pp.109.138677
[30] M. Tikkanen, M. Nurmi, S. Kangasjarvi and E. M. Aro, "Core Protein Phosphorylation Facilitates the Repair of Photodamaged Photosystem II at High Light,” Biochimica et Biophysica Acta, Vol. 1777, No. 11, 2008, pp. 14321437.

[31] S. Bellafiore, F. Barneche, G. Peltier and J. D. Rochaix, "State Transitions and Light Adaptation Require Chloroplast Thylakoid Protein Kinase STN7," Nature, Vol. 433, No. 7028, 2005, pp. 892-895. http://dx.doi.org/10.1038/nature03286

[32] R. Fristedt, A. Willig, P. Granath, M. Crèvecoeur, J. D. Rochaix and A. V. Vener, "Phosphorylation of Photosystem II Controls Functional Macroscopic Folding of Photosynthetic Membranes in Arabidopsis," Plant Cell, Vol. 21, No. 12, 2009, pp. 3950-3964. http://dx.doi.org/10.1105/tpc.109.069435

[33] J. D. Rochaix, "Role of Thylakoid Protein Kinases in Photosynthetic Acclimation,” FEBS Letters, Vol. 581, No. 15, 2007, pp. 2768-2775. http://dx.doi.org/10.1016/j.febslet.2007.04.038

[34] N. Depege, S. Bellafiore and J. D. Rochaix, "Role of Chloroplast Protein Kinase Stt7 in LHCII Phosphorylation and State Transition in Chlamydomonas," Science, Vol. 299, No. 5612, 2003, pp. 1572-1575. http://dx.doi.org/10.1126/science.1081397

[35] S. Lemeille, A. Willig, N. Depège-Fargeix, C. Delessert, R. Bassi and J. D. Rochaix, "Analysis of the Chloroplast Protein Kinase Stt7 during State Transitions,” PLOS Biology, Vol. 7, No. 3, 2009, Article ID: e1000045. http://dx.doi.org/10.1371/journal.pbio.1000045

[36] I. Carlberg, E. Rintamäki, E. M. Aro and B. Andersson, "Thylakoid Protein Phosphorylation and the Thiol Redox State," Biochemistry, Vol. 38, No. 10, 1999, pp. 31973204. http://dx.doi.org/10.1021/bi982506o

[37] A. Rokka, E. M. Aro, R. G. Herrmann, B. Andersson and A. V. Vener, "Dephosphorylation of Photosystem II Reaction Center Proteins in Plant Photosynthetic Membranes as an Immediate Response to Abrupt Elevation of Temperature," Plant Physiology, Vol. 123, No. 4, 2000, pp. 1525-1536. http://dx.doi.org/10.1104/pp.123.4.1525

[38] A. Rokka, M. Suorsa, A. Saleem, N. Battchikova and E. M. Aro, "Synthesis and Assembly of Thylakoid Protein Complexes: Multiple Assembly Steps of Photosystem II," Biochemical Journal, Vol. 388, Pt. 1, 2005, pp. 159-168. http://dx.doi.org/10.1042/BJ20042098

[39] H. L. Breitholtz, R. Srivastava, E. Tyystjärvi and E. Rintamäki, "LHC II Protein Phosphorylation in Leaves of Arabidopsis thaliana Mutants Deficient in Non-Photochemical Quenching,” Photosynthesis Research, Vol. 84, No. 1-3, 2005, pp. 217-223.

[40] P. Pesaresi, M. Pribil, T. Wunder and D. Leister, "Dynamics of Reversible Protein Phosphorylation in Thylakoids of Flowering Plants: The Roles of STN7, STN8 and TAP38," Biochimica et Biophysica Acta, Vol. 1807, No. 8, 2011, pp. 887-896. http://dx.doi.org/10.1016/j.bbabio.2010.08.002

[41] J. Kargul, M. V. Turkina, J. Nield, S. Benson, A. V. Vener and J. Barber, "Light-Harvesting Complex II Protein CP29 Binds to Photosystem I of Chlamydomonas rein- 
hardtii under State 2 Conditions,” FEBS Journal, Vol. 272, No. 18, 2005, pp. 4797-4806.

http://dx.doi.org/10.1111/j.1742-4658.2005.04894.x

[42] M. V. Turkina, J. Kargul, A. Blanco-Rivero, A. Villarejo, J. Barber and A. V. Vener, "Environmentally Modulated Phosphoproteome of Photosynthetic Membranes in the Green Alga Chlamydomonas reinhardtii,” Molecular \& Cellular Proteomics, Vol. 5, No. 8, 2006, pp. 1412-1425. http://dx.doi.org/10.1074/mcp.M600066-MCP200

[43] M. Hansson, T. Dupuis, R. Strömquist, B. Andersson, A. V. Vener and I. Carlberg, "The Mobile Thylakoid Phosphoprotein TSP9 Interacts with the Light-Harvesting Complex II and the Peripheries of Both Photosystems," Journal of Biological Chemistry, Vol. 282, No. 22, 2007, pp. 16214-16222. http://dx.doi.org/10.1074/jbc.M605833200

[44] R. Fristedt, I. Carlberg, A. Zygadlo, M. Piippo, M. Nurmi, E. M. Aro, H. V. Scheller and A. V. Vener, "Intrinsically Unstructured Phosphoprotein TSP9 Regulates Light Harvesting in Arabidopsis thaliana," Biochemistry, Vol. 48, No. 2, 2009, pp. 499-509. http://dx.doi.org/10.1021/bi8016334

[45] R. Fristedt and A. V. Vener, "High Light Induced Disassembly of Photosystem II Supercomplexes in Arabidopsis Requires STN7-Dependent Phosphorylation of CP29," PLoS ONE, Vol. 6, No. 9, 2011, Article ID: e24565. http://dx.doi.org/10.1371/journal.pone.0024565

[46] M. Pribil, P. Pesaresi, A. Hertle, H. Alexander, B. Roberto and L. Dario, "Role of Plastid Protein Phosphatase TAP38 in LHCII Dephosphorylation and Thylakoid Electron Flow," PLoS Biology, Vol. 8, No. 1, 2010, Article ID: e1000288. http://dx.doi.org/10.1371/journal.pbio.1000288

[47] A. Shapiguzov, B. Ingelsson, I. Samol, C. Andres, F. Kessler, J. D. Rochaix, A. V. Vener and M. Goldschmidt-Clermont, "The PPH1 Phosphatase Is Specifically Involved in LHCII Dephosphorylation and State Transitions in Arabidopsis," Proceedings of the National Academy of Sciences of the United States of America, Vol. 107, No. 10, 2010, pp. 4782-4787. http://dx.doi.org/10.1073/pnas.0913810107

[48] E. Rintamäki, P. Martinsuo, S. Pursiheimo and E. M. Aro, "Cooperative Regulation of Light-Harvesting Complex II Phosphorylation via the Plastoquinol and FerredoxinThioredoxin System in Chloroplasts," Proceedings of the National Academy of Sciences of the United States of America, Vol. 97, No. 21, 2000, pp. 11644-11649. http://dx.doi.org/10.1073/pnas.180054297

[49] P. Singh-Rawal, A. Jajoo, S. Mathur, P. Mehta and S. Bharti, "Evidence that $\mathrm{pH}$ Can Drive State Transitions in Isolated Thylakoid Membranes from Spinach,” Photochemical and Photobiological Sciences, Vol. 9, No. 6, 2010, pp. 830837. http://dx.doi.org/10.1039/c0pp00055h

[50] S. S. Hong and D. Q. Xu, "Light-Induced Increase in Initial Chlorophyll Fluorescence $F_{0}$ Level and the Reversible Inactivation of PS II Reaction Centers in Soybean Leaves," Photosynthesis Research, Vol. 61, No. 3, 1999, pp. 269-280. http://dx.doi.org/10.1023/A:1006357203466

[51] D. Elrad, K. K. Niyogi and A. R. Grossman, “A Major Light-Harvesting Polypeptide of Photosystem II Func- tions in Thermal Dissipation,” Plant Cell, Vol. 14, No. 8, 2002, pp. 1801-1816.

http://dx.doi.org/10.1105/tpc.002154

[52] J. P. Vainonen, M. Hansson and A. V. Vener, "STN8 Protein Kinase in Arabidopsis thaliana Is Specific in Phosphorylation of Photosystem II Core Proteins,” Journal of Biological Chemistry, Vol. 280, No. 39, 2005, pp. 3367933686. http://dx.doi.org/10.1074/jbc.M505729200

[53] J. P. Vainonen, Y. Sakuragi, S. Stael, M. Tikkanen, Y. Allahverdiyeva, V. Paakkarinen, E. Aro, M. Suorsa, H. V. Scheller, A. V. Vener and E. M. Aro, "Light Regulation of CaS, a Novel Phosphoprotein in the Thylakoid Membrane of Arabidopsis thaliana," FEBS Journal, Vol. 275, No. 8, 2008, pp. 1767-1777. http://dx.doi.org/10.1111/j.1742-4658.2008.06335.x

[54] A. V. Vener, A. Rokka, H. Fulgosi, B. Andersson and R. G. Herrmann, “A Cyclophilin-Regulated PP2A-Like Protein Phosphatase in Thylakoid Membranes of Plant Chloroplasts,” Biochemistry, Vol. 38, No. 45, 1999, pp. 1495514965. http://dx.doi.org/10.1021/bi990971v

[55] E. Rintamäki, R. Kettunen and E. M. Aro, "Differential D1 Dephosphorylation in Functional and Photodamaged Photosystem II Centers. Dephosphorylation Is a Prerequisite for Degradation of Damaged D1," Journal of Biological Chemistry, Vol. 271, No. 25, 1996, pp. 14870-14875. http://dx.doi.org/10.1074/jbc.271.25.14870

[56] I. Samol, A. Shapiguzov, B. Ingelsson, G. Fucile, M. Crèvecoeur, A. V. Vener, J. D. Rochaix and M. GoldschmidtClermont, "Identification of a Photosystem II Phosphatase Involved in Light Acclimation in Arabidopsis," Plant Cell, Vol. 24, No. 6, 2012, pp. 2596-2609. http://dx.doi.org/10.1105/tpc.112.095703

[57] J. D. Rochaix, S. Lemeille, A. Shapiguzov, I. Samol, G. Fucile, A. Willig and M. Goldschmidt-Clermont, “ Protein Kinases and Phosphatases Involved in the Acclimation of the Photosynthetic Apparatus to a Changing Light Environment," Philosophical Transactions of the Royal Society of London series B-Biological Sciences, Vol. 367, No. 1608, 2012, pp. 3466-3474.

http://dx.doi.org/10.1098/rstb.2012.0064

[58] K. J. Dietz and T. Pfannschmidt, "Novel Regulators in Photosynthetic Redox Control of Plant Metabolism and Gene Expression,” Plant Physiology, Vol. 155, No. 4, 2011, pp. 1477-1485. http://dx.doi.org/10.1104/pp.110.170043

[59] L. Lupínková and J. Komenda, “Oxidative Modifications of the Photosystem II D1 Protein by Reactive Oxygen Species: From Isolated Protein to Cyanobacterial Cells," Photochemistry and Photobiology, Vol. 79, No. 2, 2004, pp. 152-162.

[60] I. Vass, "Role of Charge Recombination Processes in Photodamage and Photoprotection of the Photosystem II Complex,” Physiologia Plantarum, Vol. 142, No. 1, 2011, pp. 6-16. http://dx.doi.org/10.1111/j.1399-3054.2011.01454.x

[61] S. I. Allakhverdiev, Y. Nishiyama, S. Takahashi, S. Miyairi, I. Suzuki and N. Murata, "Systematic Analysis of the Relation of Electron Transport and ATP Synthesis to the Photodamage and Repair of Photosystem II in 
Synechocystis,” Plant Physiology, Vol. 137, No. 1, 2005, pp. 263-273. http://dx.doi.org/10.1104/pp.104.054478

[62] N. Murata, S. Takahashi, Y. Nishiyama and S. I. Allakhverdiev, "Photoinhibition of Photosystem II under Environmental Stress,” Biochimica et Biophysica Acta (BBA)Bioenergetics, Vol. 1767, No. 6, 2007, pp. 414-421. http://dx.doi.org/10.1016/j.bbabio.2006.11.019

[63] R. Barbato, G. Friso, F. Rigoni, F. Dalla Vecchia and G. M. Giacometti, "Structural Changes and Lateral Redistribution of Photosystem II during Donor Side Photoinhibition of Thylakoids,” Journal of Cell Biology, Vol. 119, No. 2, 1992, pp. 325-335. http://dx.doi.org/10.1083/jcb.119.2.325

[64] M. Tikkanen and E. M. Aro, “Thylakoid Protein Phosphorylation in Dynamic Regulation of Photosystem II in Higher Plants," Biochimica et Biophysica Acta (BBA)Bioenergetics, Vol. 1817, No. 1, 2012, pp. 232-238. http://dx.doi.org/10.1016/j.bbabio.2011.05.005

[65] P. F. Huesgen, H. Schuhmann and I. Adamska, "The Family of Deg Proteases in Cyanobacteria and Chloroplasts of Higher Plants,” Physiologia Plantarum, Vol. 123, No. 4, 2005, pp. 413-420. http://dx.doi.org/10.1111/j.1399-3054.2005.00458.x

[66] M. Lindahl, C. Spetea, T. Hundal, A. B. Oppenheim, Z. Adam and B. Andersson, "The Thylakoid FtsH Protease Plays a Role in the Light-Induced Turnover of the Photosystem II D1 Protein,” Plant Cell, Vol. 12, No. 3, 2000, pp. 419-431.

[67] R. Fristedt, P. Granath and A. V. Vener, “A Protein Phosphorylation Threshold for Functional Stacking of Plant Photosynthetic Membranes,” PLoS ONE, Vol. 5, No.
6, 2010, Article ID: e10963. http://dx.doi.org/10.1371/journal.pone.0010963

[68] E. M. Aro, S. McCaffery and J. M. Anderson, "Photoinhibition and D1 Protein Degradation in Peas Acclimated to Different Growth Irradiances," Plant Physiology, Vol. Vol. 103, No. 3, 1993, pp. 835-843.

[69] J. M. Anderson and E. M. Aro, “Grana Stacking and Protection of Photosystem II in Thylakoid Membranes of Higher Plant Leaves under Sustained High Irradiance: An Hypothesis," Photosynthesis Research, Vol. 41, No. 2, 1994, pp. 315-326. http://dx.doi.org/10.1007/BF00019409

[70] L. Chen, H. Jia, Q. Tian, L. Du, Y. Gao, X. Miao and Y. Liu, "Protecting Effect of Phosphorylation on Oxidative Damage of D1 Protein by Down-Regulating the Production of Superoxide Anion in photosystem II Membranes under High Light,” Photosynthesis Research, Vol. 112, No. 2, 2012, pp. 141-148. http://dx.doi.org/10.1007/s11120-012-9750-9

[71] Y. E. Chen, Z. Y. Zhao, H. Y. Zhang, X. Y. Zeng and S. Yuan, "The Significance of CP29 Reversible Phosphorylation in Thylakoids of Higher Plants under Environmental Stresses,” Journal of Experimental Botany, Vol. 64, No. 5, 2013, pp. 1167-1178. http://dx.doi.org/10.1093/jxb/ert002

[72] T. K. Goral, M. P. Johnson, A. P. Brain, H. Kirchhoff, A. V. Ruban and C. W. Mullineaux, "Visualizing the Mobility and Distribution of Chlorophyll Proteins in Higher Plant Thylakoid Membranes: Effects of Photoinhibition and Protein Phosphorylation,” Plant Journal, Vol. 62, No. 6, 2010, pp. 948-959. 